\title{
Data Mining Algorithms for Pharmacovigilance
}

\author{
Kamatchi Sankar, Latha Parthiban
}

\begin{abstract}
In this paper, various data mining algorithms for pharmacovigilance is analyzed and a decision support system for hospital is proposed.. Overall analysis of adverse events of a specific drug helps in finding the potential danger of using the specific drug. Decision support system with good classification accuracy to improve its use in hospital for computer aided diagnosis by doctors is also analyzed,
\end{abstract}

Index Terms: Pharmacovigilance, Adverse event, classification accuracy, computer aided diagnosis.

\section{INTRODUCTION}

Data mining deals with extracting meaningful patterns from huge datasets using hybridized method involving computational intelligence, artificial intelligence and statistics[1]. Medical data mining deals with classification techniques using medical datasets [2] that improves the quality of computer aided of health services. Figure 1 shows the decision support system used in hospitals.

Pharmacovigilance $(\mathrm{PhV})$ aims at the best use of medicine to cure the disease[3] with no adverse effects and World Health Organization (WHO) defines it as "the science and activities relating to the detection, assessment, understanding and prevention of adverse effects or any other possible drug-related problems" [4]. PhV must "promote and protect public health by reducing burden of ADRs and optimizing the use of medicines".

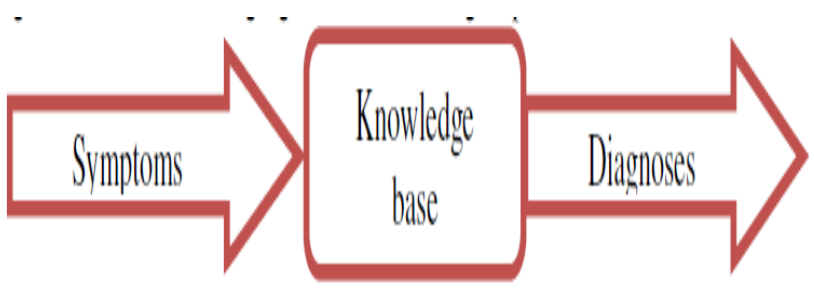

Fig. 1a Decision support system

Revised Manuscript Received on October 18, 2019.

Kamatchi Sankar, ,Research Scholar, Dept. of Computer Science \& Engg, Bharath Institute of Higher Education and Research, Tamilnadu, India,Email : sankam1309@gmail.com

Latha Parthiban,' Department Computer Science and Engineering, Pondicherry University Community College, Puducherry, India.

Email : lathaparthiban@yahoo.com

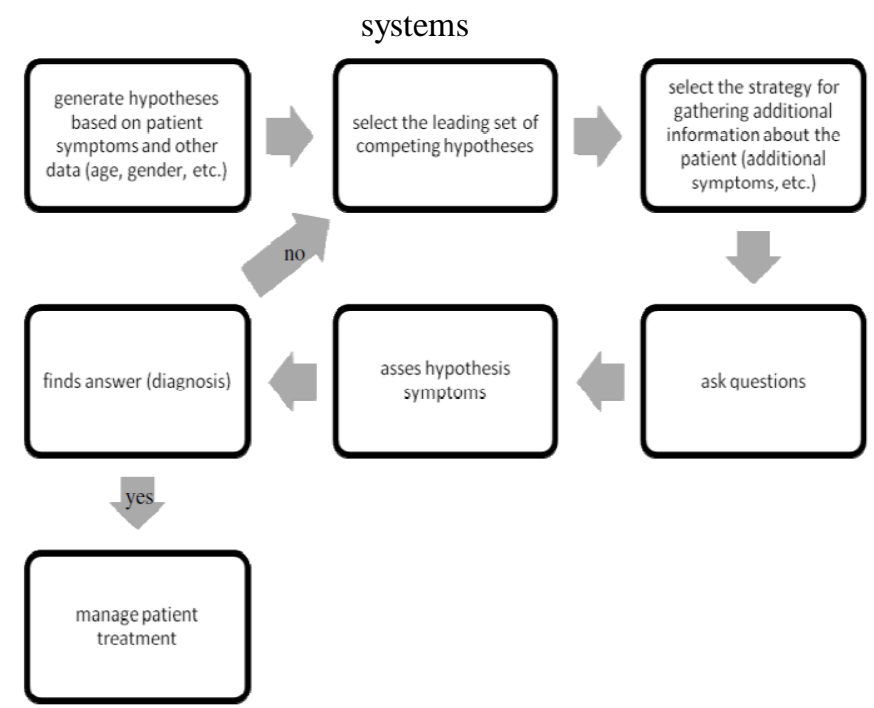

Figure 1(b) Hypothesis based decision support system

\section{PREVIOUS WORK}

Clinical Decision Support System is an approach where we give the application the required features about any specific disease in which we train the algorithm to predict whether the patient has that disease or not[5-10]. This Clinical Decision Support System plays a major role in today's busy life where time has become a major issue and also seriousness of the disease can be found immediately and necessary actions can be taken to cure the disease[11-14]. Also, in some of the cases we can avoid the unnecessary tests which are performed to find whether the disease is present or not[15-23].

A classification technique based Artificial Neural Network[24] is designed with Back Propagation Network and Cascaded Correlation Neural Network and got accuracy of $79.45 \%$. A feature selection based on Convolutional Neural Network and classification is designed based on Deep Belief Network [25-26] and got accuracy of 90\%. A feature selection based on Entropy Ensemble of Neural Networks[27] is designed with Recursive Feature Elimination and better results are achieved . Classification was based on Support Vector Machine with Recursive Feature Elimination and got an accuracy of 85.66\%[28].In [29] classification based on Deep Belief Network was designed and got accuracy of $83.9 \%$. Table 1 shows some important class of drugs and its adverse effects.

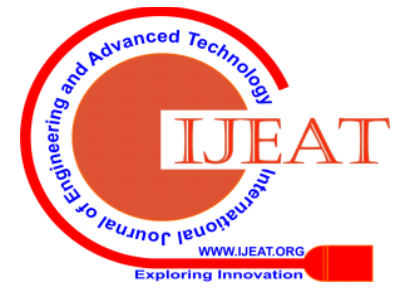


Data Mining Algorithms for Pharmacovigilance

Table 1 Drugs and its effects

\begin{tabular}{|ll|}
\hline \multicolumn{1}{|c|}{ Class of Drugs } & \multicolumn{1}{c|}{ Effects } \\
\hline Tetracycline & Poor absorbtion of tetracyclines \\
Amino glycoside & Hearing problem, kidney problem \\
Anti diabetic & Lower blood sugar \\
Warfarin & Increased risk of bleeding \\
Phenytoin & CNS and Respiratory depression \\
Barbiturates & Muscle weakness,Reduced consciousness, \\
Lithium & coma \\
Alprazalon, & Hypothermia \\
Diazepam & CNS depression, sedation \\
Warfarin & Haemorrhage \\
Methotrexate & Bone marrow suppression \\
Benzodiazepines & Sedation and Respiratory suppression \\
Ethanol & Additive CNS effect, Death \\
Predmisone & Edema \\
Theophyllines & Insomnia, seizures, restlessness \\
Miconazole & Severe hypoglycaemia \\
\hline
\end{tabular}

\section{KNOWLEDGE BASED DECISION SUPPORT SYSTEM}

Figure 2 shows the knowledge based decision support system where problem specific knowledge is used for decision making. The flowchart of choice based implementation of DSS is shown in figure 3. The usage of medical DSS in hospitals is shown in figure 4.

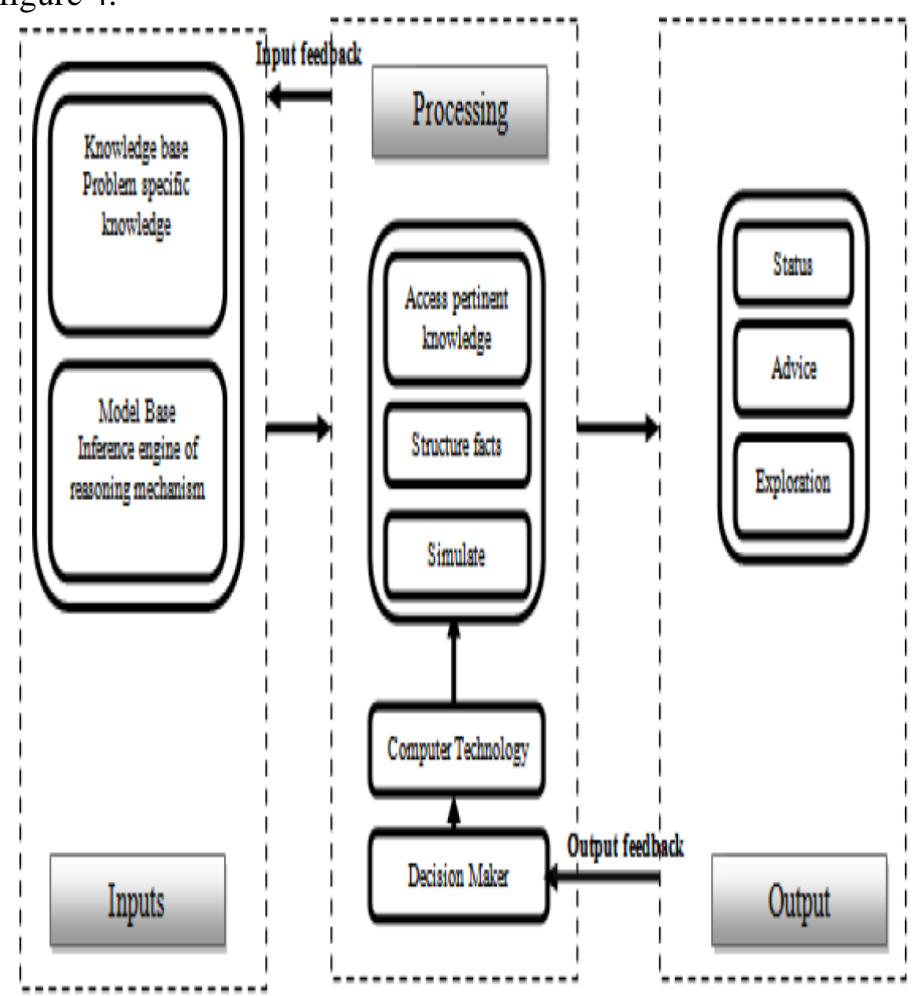

Fig 2 Knowledge based DSS

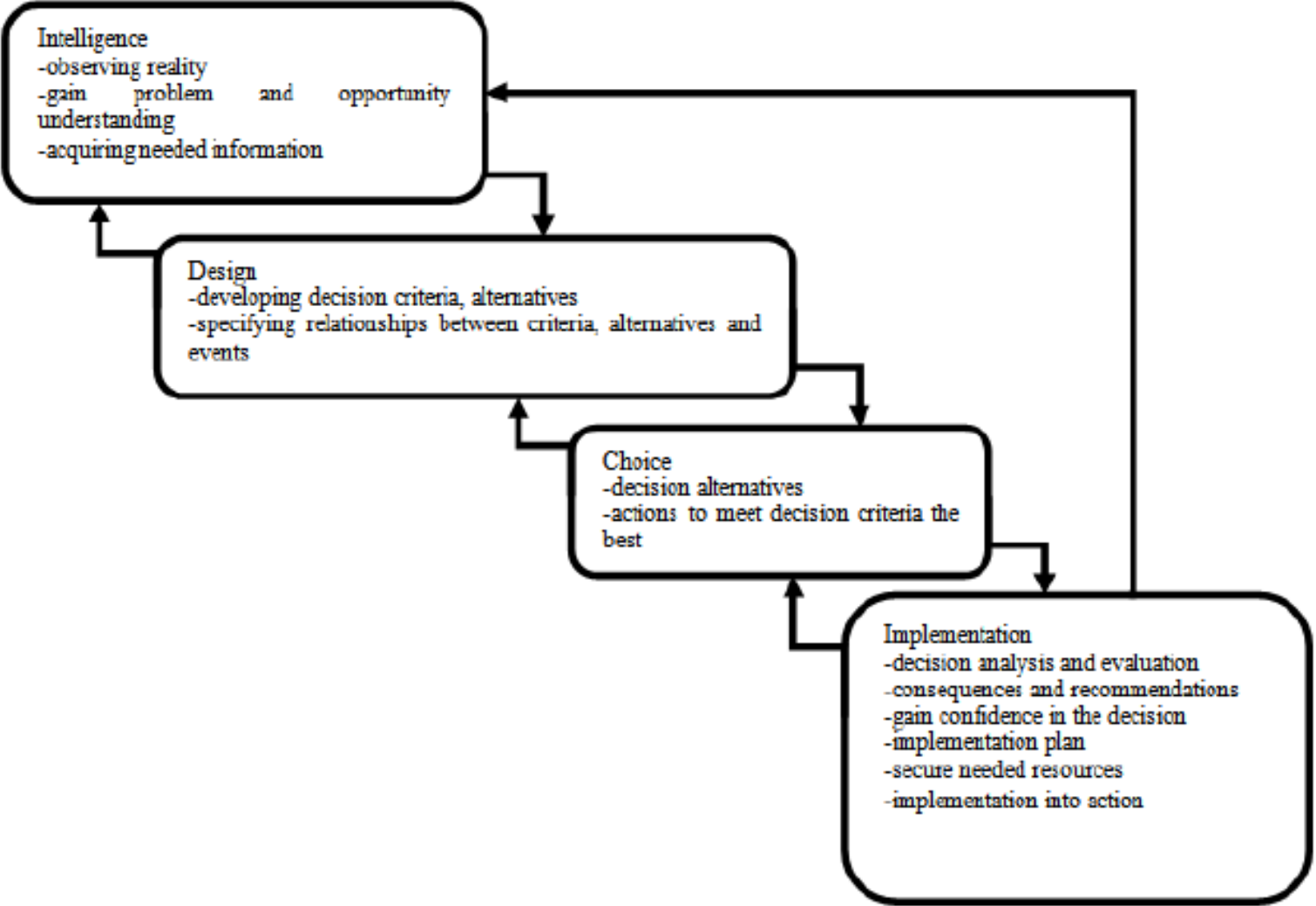

Fig 3 Implementation of DSS 


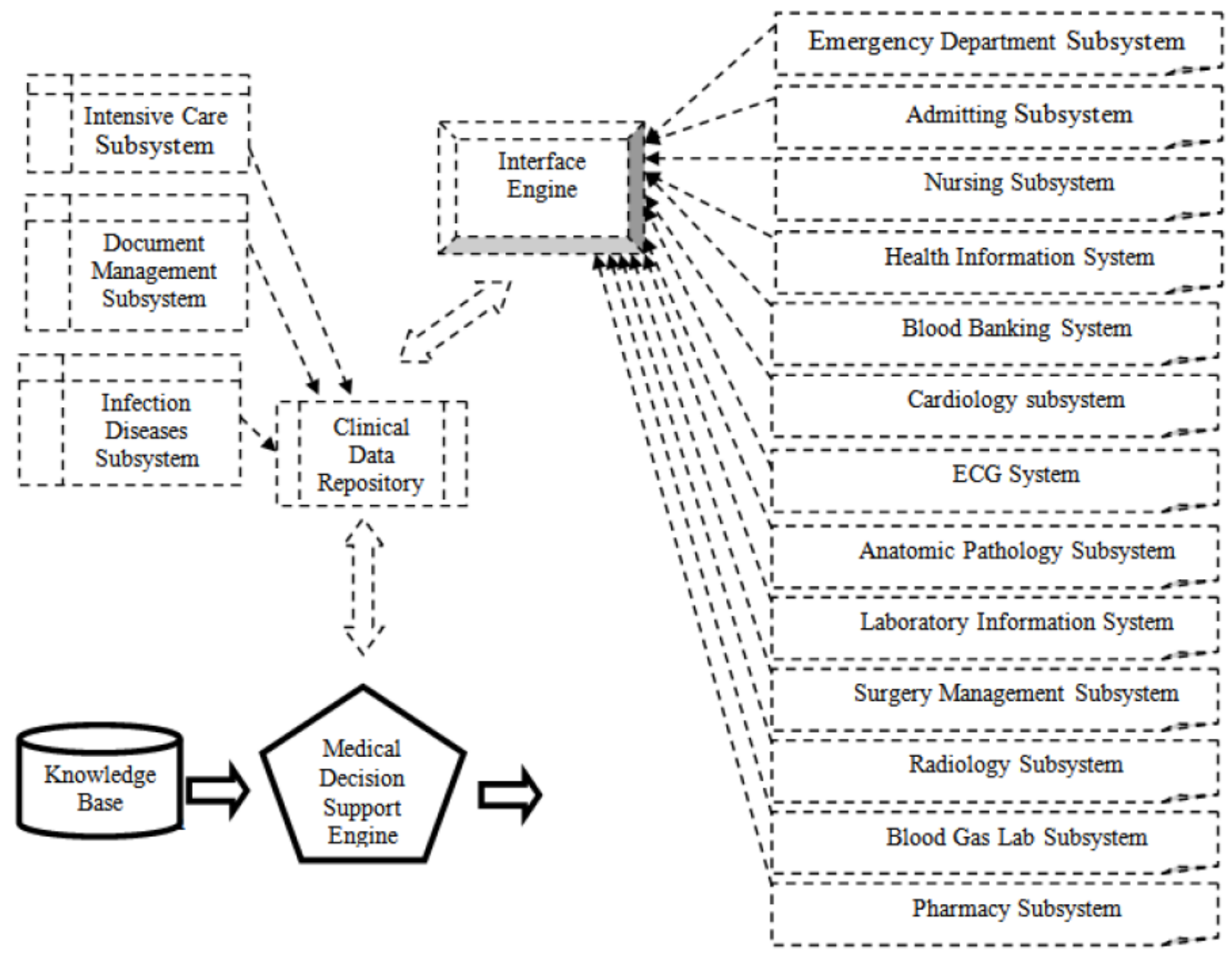

hospitals

Fig 4 DSS for

\section{DATA MINING ALGORITHMS}

Many data mining algorithms are used for Pharmacovigilance purpose and two important algorithms are analyzed in this work

Given: Sampl - the set of training examples ( $S a m p l \subseteq T, T$ is decision table), $d_{k^{-}}$the attribute which value is to be predicted by the tree, $S=\left\{s_{1}, \ldots, s_{I}\right\}$ the set of symptoms

Results: The decision tree Tree

BEGIN

1. For $1 \leq k \leq K$ compute decision frequencies freq $\left(d_{k,}, \operatorname{Sampl}\right)$

2. If all cases in $S a m p l$ belongs to the same class $d_{k}$ set the node is a leaf with associated class $d_{\mathrm{k}}$.

3. Else set as a node the most frequent class $d_{k}$, and count the classification error a of the leaf is the weighted sum of the cases in Sampl, whose class is not $d_{k}$.

4. For $s_{i}, i \in(1, I)$ count $\operatorname{Gain}\left(\operatorname{Sampl}_{,} s_{t}\right)$

5. Set $s_{g}:=s_{i}$ the attribute with the highest $\operatorname{Gatn}\left(\operatorname{Samp} \mathbb{I}_{,} s_{i}\right)$

6. If $s_{g}$ is continuous find Threshold

7. For each Sampl' in the splitting of Sampl DO 8-9

8. If $\operatorname{SampL^{r}}=\emptyset$ set as a child of $s_{t}$ is a leaf

9. Else below this new branch add the subtree $\mathrm{C} 4.5$ for $d_{\mathrm{k}}$ and $S=\left\{s_{1}, \ldots, s_{I}\right\} \backslash s_{g}$

10. Compute errors of $s_{g}$

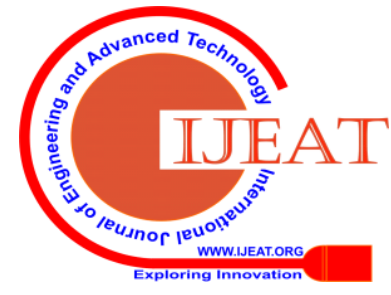




\section{Data Mining Algorithms for Pharmacovigilance}

Given: $S a m p l$ - the set of training examples ( $\operatorname{Sampl} \subseteq T, T$ is decision table), $d_{k^{-}}$the attribute which value is to be predicted by the tree, $S=\left\{s_{1}, \ldots, s_{l}\right\}$ the set of symptoms

Results: The decision tree Tree

BEGIN

1. If all examples are positive, Return the single-node tree Root, with label $=+$ END

2. If all examples are negative, Return the single-node tree Root, with label $=-$ END

3. If number of predicting attributes is empty, then Return the single node tree Root, with label $=$ most common value of the target attribute in the examples END

4. Set $s_{g}:=s_{i}$ the attribute with the highest $\operatorname{Gatn}\left(\operatorname{Sampl}, s_{i}\right)$

5. Set $s_{g}$ as a Root of the decision tree

6. For each $v \in$ Values $\left(s_{g}\right)$ DO 7-10

7. Add a new tree branch below Root, corresponding to the test $s_{g}=v_{t}$ :

8. Let examples $\left(v_{i}\right)$, be the subset of examples that have the value $v_{i}$ for $s_{g}$.

9. If examples $\left(v_{i}\right)$ is empty Then below this new branch add a leaf node with label $=$ most common target value in the examples END

10. Below this new branch add the subtree ID3 (examples $\left.\left(v_{i}\right), d_{k}, S=\left\{s_{1}, \ldots, s_{l}\right\} \backslash s_{g}\right)$ END

Return Root

\section{CONCLUSIONS}

In this paper, decision support systems used for treatment is analyzed and an architecture used in hospital for decision making is proposed. As pharmacovigilance has become an important research area as on date, some important commonly used drugs and its effects are tabulated. Data mining algorithms used for Pharmacovigilance is also analyzed. Future work will be to enhance the algorithms for effective pharmacovigilance.

\section{REFERENCES}

1. Liu V, Kipnis P, Rizk NW, Escobar GJ. Adverse outcomes associated with delayed intensive care unit transfers in an integrated healthcare system. $\mathbf{J}$ Hosp Med 2012;7: 224-30.

2. Escobar GJ, Greene JD, Gardner MN, et al. Intra-hospital transfers to a higher level of care: contribution to total hospital and intensive care unit (ICU) mortality and length of stay (LOS). J Hosp Med 2011;6:74-80.

3. Delgado MK, Liu V, Pines JM, et al. Risk factors for unplanned transfer to intensive care within 24 hours of admission from the emergency department in an integrated healthcare system. J Hosp Med 2013;8:13-9.

4. Zegers M, de Bruijne MC, Wagner C, et al. Adverse events and potentially preventable deaths in Dutch hospitals: results of a retrospective patient record review study. Qual Saf Health Care 2009;18:297-302.

5. Vlayen A, Verelst S, Bekkering GE, et al. Incidence and preventability of adverse events requiring intensive care admission: a systematic review. J Eval Clin Pract 2012;18:485-97.

6. Boulain T, Runge I, Delorme N, et al. Patients hospitalized in general wards via the emergency department: early identification of predisposing factors for death or unexpected intensive care unit admission-A historical prospective. Emerg Med Int 2014;2014:203747.

7. Bapoje SR, Gaudiani JL, Narayanan V, et al. Unplanned transfers to a medical intensive care unit: causes and relationship to preventable errors in care. J Hosp Med 2011;6:68-72. intensive care after emergency hospitalisation: risk factors and development of a nomogram for individualising risk. Resuscitation 2009;80(2):224-30.

9. Smith T, Den Hartog D, Moerman T, et al. Accuracy of an expanded early warning score for patients in general and trauma surgery wards. Brit $\mathbf{J}$ Surg 2012;99: 192-7.

10. Ludikhuize J, Smorenbueg SM, de Rooij SE, et al. Identification of deterioration patients on general wards; measurement of vital parameters an potential effectiveness of the modified early warning score. J Crit Care 2012;27: 424.e7-424.e13.

11. Petersen JA, Mackel R, Antonsen K, et al. Serious adverse events in a hospital using early warning score -what went wrong? Resuscitation 2014;85:1699-703.
8. Frost SA, Alexandrou E, Bogdanovski T, et al. Unplanned admission to

12. Gold CA, Mayer SA, Lennihan L, et al. Unplanned transfers fromhospitalwards to the neurological intensive care unit. Neurocrit Care 2015;23:159-65.

13. Calder L, Pozgay A, Riff S, et al. Adverse events in patients with return emergency department visits. BMJ Qual Saf 2015;24:142-8.

14. Dunn KL, Reddy P, Moulden A, et al.Medical record review of deaths, unexpected intensive care unit admissions, and clinician referrals: detection of adverse events and insight into the system. Arch Dis Child 2006;91:169-72.

15. Naessens JM, O'Byrne TJ, Johnson MG, et al. Measuring hospital adverse events: assessing inter-rater reliability and trigger performance of the Global Trigger Tool. Int J Qual Health Care 2010;22:266-74.

16. Lindquist M, Ståhl, M., Bate, A., Edwards, I. R., Meyboom, R. H.B. A retrospective evaluation of a data mining approach to aid finding new adverse drug reaction signals in the WHO international database. Drug Safety 2000;23(6):533-542.

17. K. Zarkogianni et al., "A review of emerging technologies for the management of diabetes mellitus," IEEE Trans. Biomed. Eng., vol. 62, no. 12 , pp. 2735-2749, Dec. 2015.

18. F. Mansourypoor and S. Asadi, "Development of a reinforcement learningbased evolutionary fuzzy rule-based system for diabetes diagnosis,' Comput. Biol. Med., vol. 91, pp. 337-352, Dec. 2017.

19. L. Bickley, Bates' Guide to Physical Examination and History-Taking, 11 th ed. Philadelphia, PA, USA: LWW, 2013.

20. A. Minutolo, M. Esposito, and G. De Pietro, “A fuzzy framework for encoding uncertainty in clinical decision-making," Knowl.-Based Syst., vol. 98, pp. 95-116, Apr. 2015.

21. M. Maniruzzaman et al., "Comparative approaches for classification of diabetes mellitus data: Machine learning paradigm," Comput. Methods Programs Biomed., vol. 152, pp. 23-34, Dec. 2017.

22. O. Biran and C. Cotton, "Explanation and justification in machine learning: A survey," in Proc. Int. Justification Conf. Artif. Intell. Workshop Explainable Artif. Intell., 2017, pp. 8-13.

23. J. M. Alonso, C. Castiello, M. Lucarelli, and C. Mencar, "Modeling interpretable fuzzy rule-based classifiers for medical decision support," in Medical Applications of Intelligent Data Analysis: Research Advancements. Hershey, PA, USA: IGI Global, 2013, pp. 1064-1081.

24. S. Guillaume and B. Charnomoridc, "Learning interpretable fuzzy inference systems with FisPro," Inf. Sci., vol. 181, no. 20, pp. 4409-4427, 2011. [9] L. A. Zadeh, "From computing with numbers to computing with words. From manipulation of measurements to manipulation of perceptions,' IEEE Trans. Circuits Syst. I. Fundam. Theory Appl., vol. 46, no. 1, pp. 105-119, Jan. 1999.

25. J. M. Alonso, A. Ramos-Soto, E. Reiter, and K. van Deemter, "An exploratory study on the benefits of using natural language for explaining fuzzy rule-based systems,', in Proc. IEEE Int. Conf. Fuzzy Syst., Jul. 2017, pp. 1-6.

26. J. M. Alonso, C. Castiello, and C. Mencar, "Interpretability of fuzzy systems: Current research trends and prospects,' in Springer Handbook of Computational Intelligence. Berlin, Germany: Springer, 2015, pp. 219-237. 
27. R. Meza-Palacios, A. A. Aguilar-Lasserre, E. L. Ureña-Bogarín, C. F. Vázquez-RodrÃguez, R. Posada-GÃşsmez, and A. Trujillo-Mata, "Development of a fuzzy expert system for the nephropathy control assessment in patients with type 2 diabetes mellitus,' Expert Syst. Appl., vol. 72, no. 1, pp. 335-343, Apr. 2017.

28. A. D. Torshizi, M. H. F. Zarandi, G. D. Torshizi, and K. Eghbali, "A hybrid fuzzy-ontology based intelligent system to determine level of severity and treatment recommendation for benign prostatic hyperplasia,' Comput. Methods Programs Biomed., vol. 113, no. 1, pp. 301-313, 2014.

29. S. El-Sappagh, M. Elmogy, and A. M. Riad, "A fuzzy-ontology-oriented case-based reasoning framework for semantic diabetes diagnosis,' Artif. Intell. Med., vol. 65, no. 3, pp. 179-208, 2015.

30. K. Vijayakumar,C.Arun,Automated risk identification using NLP in cloud based development environments,J Ambient Intell Human Computing,DOI 10.1007/s12652-017-0503-7,Springer May 2017.

31. K. Vijayakumar, Arun C, "Integrated cloud-based risk assessment model for continuous integration", International Journal Reasoning-based Intelligent Systems, Vol. 10, Nos. 3/4, 2018.

32. K. Vijayakumar, S. Suchitra and P. Swathi Shri, "A secured cloud storage auditing with empiricaloutsourcing of key updates", International Journal Reasoning-based Intelligent Systems, Vol. 11, No. 2, 2019.

\section{AUTHOR PROFILE}

Kamatchi Sankar profile which contains their education details, their publications, research work, membership, achievements, with photo that will be maximum 200-400 words.

Latha Parthiban profile which contains their education details, their publications, research work, membership, achievements, with photo that will be maximum 200-400 words. 